Documentation et bibliothèques

\title{
La recherche en histoire de la presse au Québec : bilan bibliométrique
}

\section{Jean de Bonville}

Volume 41, numéro 3, juillet-septembre 1995

URI : https://id.erudit.org/iderudit/1033235ar

DOI : https://doi.org/10.7202/1033235ar

Aller au sommaire du numéro

Éditeur(s)

Association pour l'avancement des sciences et des techniques de la

documentation (ASTED)

ISSN

0315-2340 (imprimé)

2291-8949 (numérique)

Découvrir la revue

Citer cet article

de Bonville, J. (1995). La recherche en histoire de la presse au Québec : bilan bibliométrique. Documentation et bibliothèques, 41(3), 169-172.

https://doi.org/10.7202/1033235ar

Tous droits réservés (C) Association pour l'avancement des sciences et des techniques de la documentation (ASTED), 1995
Ce document est protégé par la loi sur le droit d'auteur. L'utilisation des services d'Érudit (y compris la reproduction) est assujettie à sa politique d'utilisation que vous pouvez consulter en ligne.

https://apropos.erudit.org/fr/usagers/politique-dutilisation/ 


\title{
La recherche en histoire de la presse au Québec: bilan bibliométrique
}

\author{
Jean de Bonville \\ Département d'information et de communication \\ Université Laval
}

Au catalogue des clichés à la mode figure l'affirmation selon laquelle le Québec serait entré depuis quelques décennies dans l'ère des communications. Le syntagme «autoroute électronique» n'est ni le premier ni le dernier slogan exprimant l'idéologie de la société de l'information, dont les médias constitueraient une composante essentielle. Si le rôle de l'histoire est d'interpréter le passé à la lumière des préoccupations actuelles, nous devrions constater une abondante floraison d'écrits de nature historique portant sur les moyens de communication. Un inventaire systématique de la documentation sur la presse québécoise de 1764 à 1914 nous démontre, au contraire, que la communication demeure un domaine négligé de l'historiographie québécoise ${ }^{1}$.

Conscient de cette indifférence, nous avons conçu la bibliographie produite à la suite de cet inventaire comme une invitation aux apprentis chercheurs à s'intéresser à l'histoire de la presse, qui constitue un domaine de recherche fascinant, au confluent de toutes les préoccupations historiographiques ${ }^{2}$. Nous ne nous limitons donc pas à signaler les quelque 2000 documents répertoriés; ils sont aussi généreusement résumés et indexés. Pas moins de cinq index facilitent le repérage des documents: index des auteurs et des sujets, évidemment, mais aussi index des périodes couvertes dans les documents, index des périodiques et des monographies d'où sont tirés les documents analysés. Dans le corps de la bibliographie, le classement des références attire l'attention sur les forces, mais surtout - il faut l'admettre - les faiblesses de l'historiographie et sur ses cycles de production. En effet, sous chaque thème - nous reviendrons plus loin sur la distribution thématique - , le signalement des titres suit un ordre chronologique décrois- sant, si bien qu'à partir du dernier ouvrage paru sur ce thème, le lecteur peut parcourir, à rebours, les jalons du discours historiographique.

La bibliographie réunit deux sources très différentes de documents. Tout d'abord, des études, récentes ou anciennes, au nombre desquelles figurent évidemment plusieurs dizaines de thèses de doctorat et de mémoires de maîtrise, mais aussi des textes plus anciens, contemporains de leur objet d'étude comme les articles connus d'Arthur Buies et l'Abbé Provancher sur la presse des années 1870. La seconde catégorie de documents est constituée principalement de textes parus entre 1764 et 1914 , et dans lesquels leurs auteurs témoignent, à chaud, de leurs conditions de vie et de leur travail. Les historiens considèrent habituellement ces ècrits comme des sources imprimées.

Nous avons rangé $41 \%$ des titres dans des classes de documents que nous pouvons qualifier de scientifiques: travaux universitaires, monographies, chapitres de livres, articles de revue savante, communications, etc. ${ }^{3}$. C'est à ces travaux que nous nous intéresserons ici. Ils forment un noyau de près de 840 titres dont $23 \%$ sont des travaux d'étudiants universitaires, $16 \%$ des articles de revues savantes et le reste, des livres (11\%) ou parties de livres $(50 \%)^{4}$. Dans quelque 200 travaux de recherche d'étudiants universitaires, $60 \%$ sont des mémoires de maîtrise, $23 \%$ des thèses de doctorat, tandis que les autres sont des documents produits pour l'obtention d'un ancien diplôme terminal de premier cycle en bibliothéconomie. Considérées du point de vue de leur discipline universitaire, ces recherches se concentrent dans un nombre limité de secteurs: plus du tiers sont menées en histoire (38\%) plus du quart en lettres et littérature (28 \%) et une proportion à première vue étonnante en bibliothéconomie (14\%).

C'est à Adjutor Rivard qu'il revient d'avoir déposé, en 1915, la première thèse de doctorat au Québec portant sur la presse. II ne s'agit pas d'une thèse en histoire ni même sur l'histoire de la presse, mais d'un traité de droit sur la liberté de la presse au Canada. Comme cette thèse se situe à la limite de la période étudiée, nous l'avons retenue dans la bibliographie. Mais la première thèse de doctorat portant sur l'histoire de la presse n'a été déposée qu'en 1939, à l'Université d'Ottawa, par le frère Marie-Médéric. Elle traitait de l'influence de Voltaire sur le journalisme canadien. Elle a été suivie, en 1941, d'une seconde thèse, par Gérard Angers, sur les rapports entre presse et journalisme jusqu'en 1864. Le premier

1. Cette recherche bibliographique a été rendue possible grâce à une subvention du Conseil de recherches en sciences humaines du Canada, auquel nous exprimons notre reconnaissance.

2. De Bonville, Jean (dir.). La presse québécoise de 1764 à 1914: bibliographie analytique, Sainte-Foy: Presses de l'Université Laval, 1995.

3. Nous avons interprété généreusement les catégories, si bien que ce $41 \%$ comprend un bon nombre d'écrits qui relèvent plus de l'érudition que de la science.

4. Nous avons rangé dans la catégorie «parties de livre», les contributions individuelles dans des recueils collectifs comme des actes de colloque, les chapitres pertinents tirés de monographies portant sur d'autres sujets, et plus de 200 articles provenant du Dictionnaire biographique du Canada, lesquels constituent donc près du quart du corpus des écrits scientifiques et près de la moitié de la catégorie «parties de livres». 
mémoire de maîtrise, par Stanley Read, date de 1925, et portait sur le journalisme canadien d'expression anglaise de la seconde moitié du $19^{e}$ siècle. À partir de 1939 , il ne se passe aucune année sans qu'une recherche universitaire sur la presse des $18^{\circ}$ et $19^{\circ}$ siècles ne soit déposée, la moyenne étant de 3 thèses ou mémoires par année. La décennie de 1966 à 1976 est caractérisée par une productivité deux fois plus élevée, en partie, sans doute, attribuable à la vogue que connaît à cette époque l'étude des idéologies, dont la presse constitue le principal support.

La provenance des textes scientifiques est diversifiée, et la variété des auteurs est très grande. Si l'on exclut les articles du Dictionnaire biographique du Canada, auquel certains auteurs ont collaboré généreusement, rares sont les personnes qui comptent à leur actif plus de cinq textes sur l'histoire de la presse. L'auteur le plus prolifique est, sans contredit, Séraphin Marion, dont la production s'étend sur plus de trente ans, de la fin des années 1930 au début des années 1970 . Son oeuvre se répartit dans un grand nombre de revues, mais il a aussi publié plusieurs monographies sur les débuts du journalisme québécois dans la collection Lettres canadiennes d'autrefois. Par ailleurs, si l'on en juge par les publications, aucun chercheur ne semble s'être fait une spécialité de l'histoire de la presse.

Aucun périodique québécois ne s'intéresse de manière particulière à l'histoire de la presse. La seule revue savante en communication, Communication Information, n'a publié aucun article sur la presse des $18^{e}$ et $19^{\circ}$ siècles ${ }^{5}$. Si l'on exclut le Bulletin des recherches historiques, dont les articles sont, sauf exceptions, très courts, lès publications en série les plus fécondes sont les Mémoires et comptes rendus de la Société royale du Canada, d'où proviennent 27 références, les Cahiers des dix (14 références), la Revue d'histoire de l'Amérique française (12), les rapports de la Société canadienne d'histoire de l'Église catholique (10), etc. Quelques revues d'histoire régionale, que nous ne rangeons pas, cependant, dans la catégorie des écrits scientifiques, s'intéressent assez régulièrement au journalisme ou à la presse. C'est le cas notamment de la Revue d'histoire du
Bas-Saint-Laurent (11 références) et de Saguenayensia (10 références).

Nous avons divisé le siècle et demi qui va de 1764 à 1914 en quatre périodes d'inégale longueur correspondant à chacun des régimes politiques qu'a connus le Québec: à savoir le régime de la «Province of Quebeç», de 1764 à 1791 , celui du Bas-Canada, de 1791 à 1839, le régime du Canada-Uni, de 1840 à 1867, et enfin la Confédération canadienne de laquelle fait partie la province de Québec, à partir de 1867. La dernière période est la plus longue et la plus prolifique en ce qui concerne la création de journaux. II n'est donc pas étonnant que $57 \%$ des écrits scientifiques traitent de cette période. II est question de la presse du Canada-Uni dans $46 \%$ des écrits, de la presse bascanadienne, dans $31 \%$, et de la presse de 1764 à 1791 , dans $13 \%$ des recherches ${ }^{6}$. Au premier coup d'oeil, cette répartition semble équilibrée, mais si l'on considère le nombre moyen de journaux ou de revues créés à chaque année et la durée des périodes, la presse des trois premières décennies semble privilégiée: alors qu'elle compte moins de dix titres de journaux, $13 \%$ des écrits scientifiques en traitent. En revanche, les textes non scientifiques présentent une ventilation plus

che dans ce domaine porte sur l'une ou l'autre des facettes de la question suivante: «qui dit quoi, à qui, comment et avec quels moyens, dans quel but et avec quel effet?». Nous avons posé chacun de ces éléments dans l'ordre logique de la production des messages et établi six sections thématiques. Une section, qui correspond au "qui» de la formule de Lasswell, est consacrée à la vie, à l'oeuvre, aux conditions de travail et d'existence des artisans de la presse, c'est-à-dire des journalistes, éditeurs, typographes ou imprimeurs. La section suivante rassemble des écrits portant sur les conditions de fabrication de la presse et répondant à la question «comment et avec quels moyens». Deux sections, la première sur le contenu de la presse et la seconde sur la propriété intellectuelle et le contrôle politique et clérical de la presse, correspondent à la question «quoi». La section sur le public de la presse contient les documents répondant à la question «à qui», tandis que la section sur le rôle et l'influence de la presse est consacrée à la question «dans quel but et avec quel effet». Une section, intitulée "généralités», regroupe les ouvrages de référence et les écrits qui portent sur l'ensemble des thèmes en question ou un grand nombre de ceux-ci.

\section{Thèmes traités dans les écrits scientifiques et les autres écrits}

Thèmes des recherches

Généralités

Artisans de la presse

Conditions de production

Contenu de la presse

Propriété intellectuelle et contrôle

Public de la presse

Rôles et effets de la presse
Écrits scientifiques

$18 \%$
$51 \%$
$4 \%$
$21 \%$
$4 \%$
$1 \%$
$1 \%$
Autres écrits

$14 \%$
$26 \%$
$15 \%$
$16 \%$
$15 \%$
$8 \%$
$6 \%$

fidèle au dynamisme démographique (nombre de naissances de publications) de chaque période: il n'y est question des journaux du $18^{\circ}$ que dans $3 \%$ des textes, tandis que les trois périodes suivantes sont traitées respectivement dans $18 \%$, $22 \%$ et $74 \%$ des textes.

Nous avons aussi tenu compte du sujet des documents et avons regroupé les notices bibliographiques sous six thèmes inspirés de la formule d'Harold Lasswell, un des pionniers de la recherche en communication, pour qui toute recher-
Ce plan de classement (Tableau cidessus) nous servira à ventiler les travaux

5. Nous n'y avons repéré qu'une note concernant une exposition sur l'histoire de la photo imprimée au Québec.

6. Le total excède $100 \%$. Cela s'explique du fait que les auteurs peuvent traiter de plus d'une période dans une même publication. Nous avons tenu compte de toutes les périodes abordées dans les publications. Si nous additionnons les périodes couvertes dans chacun des 2030 écrits signalés, nous obtenons la somme de 2650 , ce qui veut dire qu'en moyenne chaque texte traite de 1,3 période différente! 
de recherche en fonction de leur thème. Ce tableau présente le pourcentage des écrits de nature scientifique, classés sous chacune des rubriques, et, en regard, les données concernant les autres documents signalés dans la bibliographie.

Les publications des chercheurs se concentrent dans trois sections, qui, à elles seules, rassemblent $90 \%$ du total. La section "généralités» comporte audelà de 80 bibliographies: répertoires de périodiques, index de publications, etc. De ce nombre font partie plusieurs bibliographies, genre très en vogue auprès des étudiants en bibliothéconomie de l'Université de Montréal entre le début des années 1940 et à la fin des années 1950 . Ces travaux, souvent de modeste envergure, mais parfois volumineux, signalent les oeuvres d'un journaliste et les écrits s'y rapportant. L'utilité des quelques index de périodiques - nous les avons dépouillés systématiquement pour établir notre bibliographie - fait regretter qu'il y en ait si peu: on rêve du jour où le chercheur disposera d'une indexation rétrospective des principales publications québécoises... Dans la même section «généralités» figurent bon nombre de synthèses portant soit sur une période, sur un thème, ou sur un périodique. Cependant, si l'on fait exception des livres de Jean-Paul de Lagrave, superficiels et partiaux, force est de constater que nous ne disposons d'aucun ouvrage de synthèse sur l'histoire de la presse.

C'est dans la section suivante, consacrée aux artisans de la presse, que se trouvent concentrés plus de la moitié des travaux de recherche. II s'agit évidemment de biographies, dont notamment les quelque 200 articles tirés du Dictionnaire biographique du Canada, mais aussi d'analyses de la pensée et de l'oeuvre de journalistes importants. La presque totalité des travaux de recherche rangés dans cette section portent sur des individus : seulement $4 \%$ des textes traitent des conditions de travail et d'existence des journalistes en tant que groupe. Par ailleurs, les figures de journaliste sont beaucoup plus nombreuses que celles des imprimeurs ou des éditeurs: sans doute cette répartition est-elle conforme à la réalité. En effet, la fonction d'imprimeur était beaucoup plus stable que celle de journaliste; en outre, on dénombre, en général, plusieurs journalistes à l'oeuvre dans les publications sortant des presses d'un seul imprimeur: pour un Duvernay, combien de Gosselin ou de Leblanc de Marconnay? Si l'on exclut les articles du Dictionnaire biographique, qui comptent sur autant d'individus, un nombre limité de personnalités justifient la majorité des publications. Ce sont les Asselin, Aubin, Bidaud, Bourassa, Buies, Fournier, Parent, Tardivel, etc.

Le journalisme est une forme de discours, forme privilégiée parce que saisie par la presse et, conséquemment, transmissible. II n'est pas étonnant qu'une grande partie des recherches portent sur ce discours: sur sa forme littéraire, mais surtout sur ses thèmes culturels ou sociopolitiques et sur son idéologie. En effet, $21 \%$ des textes de recherche réfléchissent vers un autre texte, journalistique celui-là. En revanche, les autres composantes de la formule de Lasswell suscitent peu l'intérêt des chercheurs. Les conditions de production de la presse, c'est-à-dire la collecte, la transmission ou le traitement de l'information et le financement ou la gestion de la presse n'attirent que $4 \%$ des recherches. Une proportion semblable est consacrée à la propriété intellectuelle et au contrôle, politique ou clérical, du contenu des journaux. La rubrique "public de la presse» rassemble des documents sur les conditions socioculturelles du public, sur les habitudes de lecture et sur la transmission des journaux. Cette section et la suivante, qui traite du rôle et des effets de la presse, n'occupent chacune que $1 \%$ de l'ensemble des recherches répertoriées.

En regard, les autres récits (c'est-àdire les écrits non scientifiques), dont un grand nombre sont contemporains de la période sur laquelle porte la bibliographie, se répartissent de manière beaucoup plus équilibrée. L'intervalle entre les deux catégories extrêmes, qui était de 50 dans le cas des recherches $(51 \%-1 \%)$, n'est que de 20 dans les autres écrits (26 $\%-6 \%)$. La section sur les artisans de la presse compte le plus grand nombre de titres, mais, à la différence des textes de recherche, ici, plus du quart des documents portent sur les conditions collectives d'existence et de travail des artisans de la presse. Trois sections présentent des proportions presque semblables: ce sont celles qui portent sur les conditions de production de la presse, sur le contenu de la presse et sur la propriété intellectuelle et le contrôle de la presse. Les deux autres sections, portant sur le public et sur le rôle de la presse, suivent avec, elles aussi, des proportions semblables, quoique deux fois moindres que les précédentes. Aucune catégorie n'occupe une fraction négligeable comme c'est le cas en ce qui concerne les publications de recherche.

Quelles constatations ces données bibliométriques inspirent-elles? Tout d'abord, elles dénotent une tendance très lourde en faveur de l'étude des individus, journaux aussi bien que journalistes. À considérer ces chiffres, l'histoire et l'histoire littéraire se révèlent des sciences fortement idiographiques. L'attrait pour le particulier, universel dans ces disciplines, est ici renforcé par la nature des sources. En effet, la disponibilité des journaux et la relative homogénéité de la production littéraire des principaux journalistes agissent comme des aimants sur la recherche. À une science théoriquement sous-développée, ils apparaissent comme autant de sujets de recherche prédéfinis, jouissant d'une existence objective.

La contrepartie de ce constat s'impose d'elle-même. Les sujets qui exigent du chercheur une certaine construction conceptuelle, même minime, sont victimes d'un manque d'intérêt systématique. Pourtant, aucune justification théorique n'explique les disproportions observées dans la ventilation thématique des recherches. Les conditions matérielles d'existence des journalistes seraient-elles réellement moins importantes que leur discours? Et si ce discours était étroitement dépendant de ces conditions? Les conditions socioculturelles du public auraient-elles moins d'influence sur le contenu de la presse que la personnalité des journalistes? L'idéologie de la presse serait-elle indépendante de ses effets? Ces questions interpellent les choix épistémologiques et méthodologiques faits, a posteriori, par l'historiographie québécoise du fait des initiatives individuelles des chercheurs. À l'évidence, la recherche québécoise sur la presse demeure singulièrement dépourvue devant des sujets qui exigent un certain effort de 
problématisation. Les historiens semblent réticents à construire leurs sujets de recherche. Ils préfèrent des sujets qui s'imposent à eux et dont l'existence est objective, comme des personnes ou des journaux. D'autre part, les historiens semblent buter sur le contenu manifeste des journaux et ils ne vont guère au-delà. IIs hésitent à soumettre le texte journalistique à d'autres instruments analytiques que ceux qui relèvent de la stylistique et de la sémantique pour ne pas dire de la thématique. II ne leur vient pas souvent à l'esprit, par exemple, d'utiliser les articles de journaux pour mesurer la vitesse de transmission de l'information ou encore les annonces publicitaires pour analyser la structure de financement de la presse. Ici, d'après nos données, absence de perspective théorique se combine avec impuissance méthodologique.

Ce jugement est-il trop sévère? La répartition des écrits non scientifiques répertoriés démontre l'existence d'une quantité appréciable de textes d'époque portant sur toutes les modalités de la proposition de Lasswell. Le matériau existe donc, mais tout se passe comme si les historiens étaient devenus myopes. Pre- nons deux cas parmi plusieurs. C'est par centaines - nous n'avons retenu que les exemples les plus représentatifs dans notre bibliographie - qu'on dénombre les doléances des éditeurs à l'endroit de leurs abonnés mauvais payeurs: à quand une étude sur les conditions financières de l'abonnement aux journaux? De même, combien d'entrefilets sur les modalités et les difficultés de la livraison postale, sur les retards dans l'acheminement des journaux, sur la mauvaise foi des maîtres de poste: les relations entre la presse et la poste sont aussi un sujet complètement négligé.

Notre bibliographie s'arrête en 1914, mais nous pensons que la recherche québécoise sur la presse du $20^{\mathrm{e}}$ valorise les mêmes thèmes et sous-estime les mêmes aspects ${ }^{7}$. Un renouvellement des perspectives et un enrichissement des problématiques nous semblent donc s'imposer. Les historiens et les spécialistes des autres sciences dites idiographiques n'y parviendront pas s'ils demeurent prisonniers d'une perspective axiologique en vertu de laquelle ils concentrent leur attention, comme ils l'ont fait jusqu'à maintenant, sur les journalistes et les publications qu'ils jugent remarquables. Au contraire, les historiens devraient privilégier les organisations, les processus, les relations sociales, etc., et s'inspirer de disciplines qui en ont fait leur objet spécifique, notamment les sciences de la communication. II est temps que la presse cesse de n'être qu'une source historique et devienne un objet de recherche en tant que telle. Nous attendons une histoire sociale de la presse.

7. Cette bibliographie a été entreprise sous les auspices' du Réseau québécois d'information sur la communication et s'est terminée sous celles du Groupe de recherche en histoire des médias au Québec. Le GRHIMEQ projette de mettre à jour la bibliographie et de la compléter en y ajoutant la documentation concernant le $20^{\circ}$ siècle. La bibliographie est aussi disponible sur support informatique. Les personnes ou institutions intéressées à se la procurer peuvent entrer en communication avec l'auteur de ce texte à l'adresse suivante: GRHIMEQ, Département d'information et de communication, Université Laval, SainteFoy, G1K 7P4. De même, toute personne qui désire collaborer aux projets, bibliographiques ou autres, du GRHIMEQ, est priée de s'adresser à l'auteur.

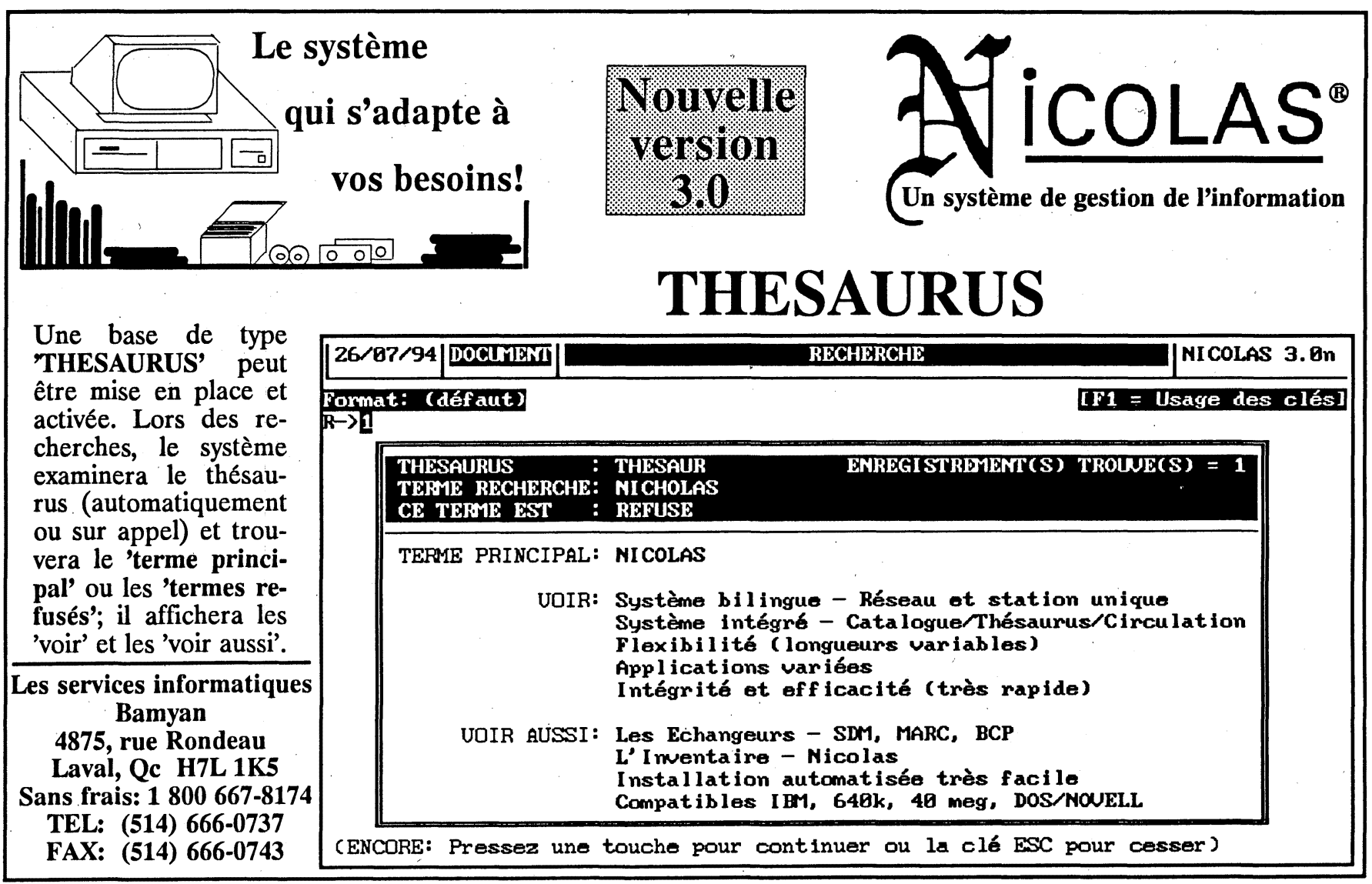

